\title{
Evaluating the effect of tumor size on survival and its prognostic significance among gastric cancer patients
}

\author{
Orhan Uzun ${ }^{1}$, Aziz Serkan Senger ${ }^{1}$, Selçuk Gülmez ${ }^{1}$, Sinan Ömeroğlu ${ }^{1}$, Cem Batuhan \\ Ofluoğlu ${ }^{1}$, Ayhan Öz ${ }^{1}$, Erdal Polat ${ }^{1}$, Mustafa Duman ${ }^{1}$ \\ ${ }^{1}$ UNIVERSITY OF HEALTH SCIENCES KARTAL KOSUYOLU HIGHER SPECIALTY TRAININ AND RESEARCH HOSPITAL, GASTROENTEROLOGY SURGERY CLINIC, ISTANBUL, TURKEY
}

Purpose. This study investigates the prognostic significance of tumor size and its effect on survival among patients undergoing gastrectomy and D2 lymph node dissection due to gastric cancer. Materials and Methods. The clinicopathological characteristics of 320 patients who were operated due to gastric cancer between November 2006 and September 2019 were assessed retrospectively, of which 271 were included in the present study. A receiveroperating characteristic curve (ROC) analysis was carried out to identify the tumor size cut-off value. Patients were divided into small-size and large-size tumor groups. Clinicopathological characteristics were assessed using Chisquare and Mann-Whitney U tests, while survival was assessed with a KaplanMeier log-rank test. Results. The cut-off gastric cancer tumor size value was calculated as $4.75 \mathrm{~cm}$. A statistical difference was noted in the tumor depth of wall invasion $(p<0.001)$, the number of positive lymph nodes removed $(\mathrm{p}<0.001)$, vascular invasion $(\mathrm{p}=0.001)$ and perineural invasion $(\mathrm{p}=0.001)$ of the two groups. Survival was poorer in patients with large-size tumors than in those with small-size tumors (62 months vs. 88 months, respectively; $\mathrm{p}<0.001)$, and tumor size was associated with wall invasion depth $(\mathrm{p}<0.001)$ and Borrmann's classification $(\mathrm{p}=0.002)$. A univariate analysis revealed tumor size to be a prognostic factor for survival $(\mathrm{p}=0.001)$, while no such finding could be established in a multivariate analysis $(\mathrm{p}=0.637)$. Conclusion. Tumor size is a prognostic marker for gastric cancer, and a preoperative assessment in this regard may suggest neoadjuvant therapy.
\end{abstract}

\section{Introduction}

Gastric cancer is the sixth most common type of cancer around the world, and among the leading causes of cancer death [1]. Aside from in Asian countries such as Japan and South Korea, gastric cancer generally presents at an advanced stage [2]. Early gastric cancer is typically treated using endoscopic methods, while the treatment for advanced stage gastric cancer is surgical resection and D2 lymph node dissection, along with pre- or post-operative therapies under a multidisciplinary approach [3]. The 5year survival rate is above $90 \%$ in early gastric cancer with the mentioned approaches, but drops to as low as 30\% depending on the stage [2].

Several prognostic indicators of gastric cancer have been identified, with the most indicative being lymph involvement and wall invasion depth, determined from postoperative pathological specimens [4,5]. Other postoperative histopathological prognostic factors include venous invasion, perineural invasion, surgical margins, peritoneal cytology and tumor size [6,7]. Tumor size is the main prognostic indication of certain solid organ tumors, such as those of the breast and liver, but is absent from the tumor, node and metastasis (TNM) staging system for gastric cancer [8]. There have been studies supporting the prognostic significance of tumor size in gastric cancer, while others make no such observation [8].

In the present study we investigate the prognostic significance of tumor size, measured postoperatively, among patients operated due to gastric cancer.

\section{Materials and Methods}

A retrospective review was made of 320 patients, who were operated due to gastric cancer in the Health Sciences University Kartal Koşuyolu High Specialty Educational and Research Hospital in Turkey, between November 2006 
and September 2019. Patient details were accessed from clinical records and pathology reports. The assessment date for the survival analysis was accepted as December 31, 2019. D2 lymph node dissections were performed in line with the recommendations of the Japanese Research Society for the Study of Gastric Cancer (JRSSG) [3], and the Tumor, Node, Metastasis (TNM) classification system devised by the American Joint of Committee on Cancer (AJCC) ( (th $^{\text {th }}$ Edition, 2018) was applied for staging [9]. Of the total, 15 patients with a positive peritoneal cytology, 10 patients with liver metastasis identified during surgery, six patients with a positive distal or proximal surgical margin, 12 patients who died within 60 days of surgery and six patients with a failed tumor size measurement were excluded from the study. The study subsequently included 271 patients. Surgery-related complications were considered as those occurring within the first 30 days following surgery.

\section{Statistical Analysis}

The normality of numerical variables was analyzed using a Kolmogorov-Smirnov test, revealing a non-normal distribution based on $\mathrm{p}<0.05$, and so median (IQR) values were used. Categorical variables were expressed as numbers and percentages. The optimal cut-off value for tumor size was established based on the results of a receiver-operating characteristic (ROC) curve analysis, and patients were divided into two groups according to the cut-off value.

A Chi-square test, a Fisher's exact test and a MannWhitney $U$ test were used to determine any statistical differences between categorical variables. Risk factors for tumor size were examined with a Binary Logistic regression analysis. The survival of the two groups was analyzed with a Kaplan-Meier test, and a log-rank test was used to identify any difference. The prognostic factors affecting overall survival were examined with a univariate and multivariate stepwise Cox regression analysis, and the statistical assessment was carried out using the SPSS 21 software package. A $p$ value of $<0.05$ was considered statistically significant.

\section{Results}

Among the 271-person sample, tumor size ranged from 0.4 to $18.0 \mathrm{~cm}$, with a median of $4.50 \mathrm{~cm}$ and a mean of $5.15 \pm 2.76 \mathrm{~cm}$ (Figure 1A). The optimal tumor size was established using the receiver-operating characteristic curve (ROC) method. The optimal cut-off value for tumor size was $4.75 \mathrm{~cm}$, with a sensitivity of $62 \%$ and a specificity of $60 \%$ (Figure 1B). The patients were divided into two groups based on tumor size (small-size tumor $\leq 4.75 \mathrm{~cm}$; large-size tumor $>4.75 \mathrm{~cm}$ ).

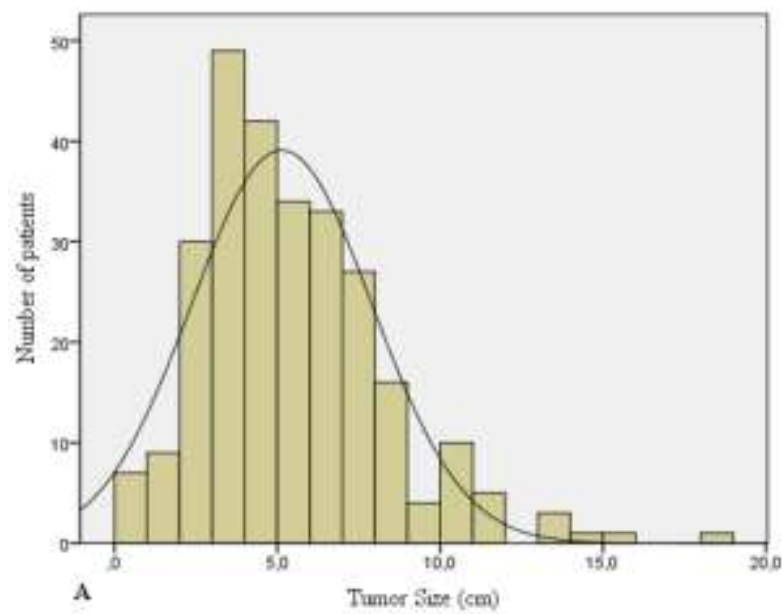

Figure 1. (A) Distribution of patients by tumor size.

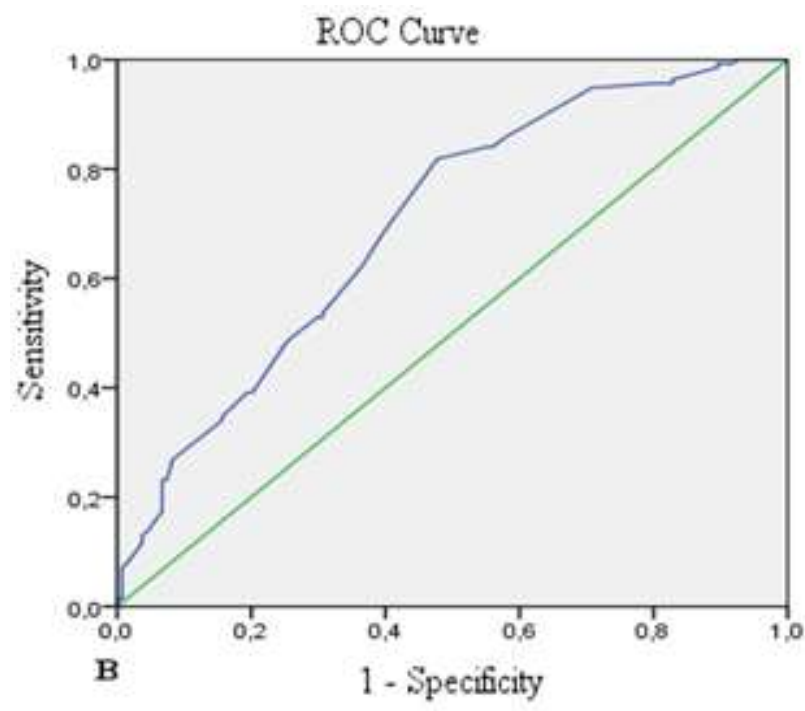

Figure 1. (B) Receiver-operating characteristic curve (ROC) for tumor size (area under the curve $=0.699$, $\mathrm{p}=0.000$ ).

A comparison of the clinicopathological characteristics of the two groups revealed statistical differences in wall invasion depth $(p<0.001)$, stage $(p<0.001)$, the number of positive lymph nodes removed $(\mathrm{p}<0.001)$, vascular invasion $(\mathrm{p}=0.001)$ and perineural invasion $(\mathrm{p}=0.001)$ (Table 1). Most of the patients in the large-size tumor group were at stage 3 based on a wall invasion depth of T3 or $\mathrm{T} 4$, and an $\mathrm{N}$ stage of $\mathrm{N} 3 \quad(38.2 \%$ and $68.1 \%$, respectively). Vascular invasion and perineural invasion were also more common in this group.

The risk factors for tumor size were examined with univariate and multivariate logistic regression analyses (Table 2), revealing risk factors of wall invasion depth $(\mathrm{p}<0.001), \mathrm{N}$ stage $(\mathrm{p}<0.001)$, vascular invasion $(\mathrm{p}=0.001)$ and perineural invasion $(\mathrm{p}=0.001)$, according to the univariate analysis, while ulcerovegetative type according to Borrmann's classification $(\mathrm{p}=0.002)$ and wall invasion depth $(p<0.001)$ were the risk factors identified in the multivariate analysis. 
Table 1. Clinicopathological features according to tumor size

\begin{tabular}{|c|c|c|c|c|c|c|}
\hline & & $\begin{array}{r}\text { Small } \\
\quad(\leq 4\end{array}$ & & $\begin{array}{l}\text { Large t } \\
\qquad>4 .\end{array}$ & & \\
\hline & & $\mathrm{n}$ & $\%$ & $\mathrm{n}$ & $\%$ & $\mathrm{p}$ value \\
\hline & Male & 90 & $66.2 \%$ & 94 & $69.6 \%$ & $0=5$ \\
\hline Gentact & Female & 46 & $33.8 \%$ & 41 & $30.4 \%$ & \\
\hline & Upper & 33 & $24.3 \%$ & 31 & $23.0 \%$ & \\
\hline Location & Middle & 27 & $19.9 \%$ & 39 & $28.9 \%$ & 0.212 \\
\hline & Distal & 76 & $55.9 \%$ & 65 & $48.1 \%$ & \\
\hline Neondiuyont the & No & 114 & $83.8 \%$ & 111 & $82.2 \%$ & 0726 \\
\hline Neoadjuvant tnerapy & Yes & 22 & $16.2 \%$ & 24 & $17.8 \%$ & 0.120 \\
\hline & Polypoid & 16 & $12.3 \%$ & 17 & $13.2 \%$ & \\
\hline clascification & Ulcerovegetative & 29 & $22.3 \%$ & 42 & $32.6 \%$ & 0.145 \\
\hline & Ulcero-infiltrative & 85 & $65.4 \%$ & 70 & $54.3 \%$ & \\
\hline Lauren's & Intestinal & 48 & $35.3 \%$ & 41 & $30.4 \%$ & 0380 \\
\hline Classification & Diffuse & 88 & $64.7 \%$ & 94 & $69.6 \%$ & 0.500 \\
\hline Tyne of Surgery & Subtotal & 77 & $56.6 \%$ & 63 & $46.7 \%$ & 0101 \\
\hline 1уре оi suigery & Total & 59 & $43.4 \%$ & 72 & $53.3 \%$ & 0.10 \\
\hline & $\mathrm{T} 1$ & 28 & $20.6 \%$ & 3 & $2.2 \%$ & \\
\hline Denth of inyacion & $\mathrm{T} 2$ & 21 & $15.4 \%$ & 9 & $6.7 \%$ & $\mathrm{n}<0001 * *$ \\
\hline Deptin or intvasion & $\mathrm{T} 3$ & 61 & $44.9 \%$ & 56 & $41.5 \%$ & $p<0.001$ \\
\hline & $\mathrm{T} 4$ & 26 & $19.1 \%$ & 67 & $49.6 \%$ & \\
\hline & Stage I & 35 & $25.7 \%$ & 9 & $6.7 \%$ & \\
\hline Stage & Stage II & 49 & $36.0 \%$ & 34 & $25.2 \%$ & $\mathrm{p}<0.001 * *$ \\
\hline & Stage III & 52 & $38.2 \%$ & 92 & $68.1 \%$ & \\
\hline Vascular Inyacion & Negative & 67 & $49.6 \%$ & 40 & $29.6 \%$ & $0001 *$ \\
\hline vascuial illvastonti & Positive & 68 & $50.4 \%$ & 95 & $70.4 \%$ & 0.001 \\
\hline Perineural Inyacion & Negative & 60 & $44.8 \%$ & 34 & $25.2 \%$ & 0001 \\
\hline 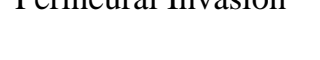 & Positive & 74 & $55.2 \%$ & 101 & $74.8 \%$ & 0.001 \\
\hline Com & No & 100 & $73.5 \%$ & 98 & $72.6 \%$ & 62 \\
\hline Compintations & Yes & 36 & $26.5 \%$ & 37 & $27.4 \%$ & 002 \\
\hline & & Med & & Media & & \\
\hline Age & & 60 & & 32( & & 0.196 \\
\hline $\begin{array}{l}\text { Total number of lymph } \\
\text { nodes }\end{array}$ & & 22 & & 26( & & 0.066 \\
\hline $\begin{array}{l}\text { Number of positive } \\
\text { lymph nodes }\end{array}$ & & & & 4( & & $\mathrm{p}<0.001 * *$ \\
\hline Length of hospital stay & & & & 10 & & 0.691 \\
\hline
\end{tabular}


Table 2. Logistic Regression Analysis of the Risk Factors for Tumor Size

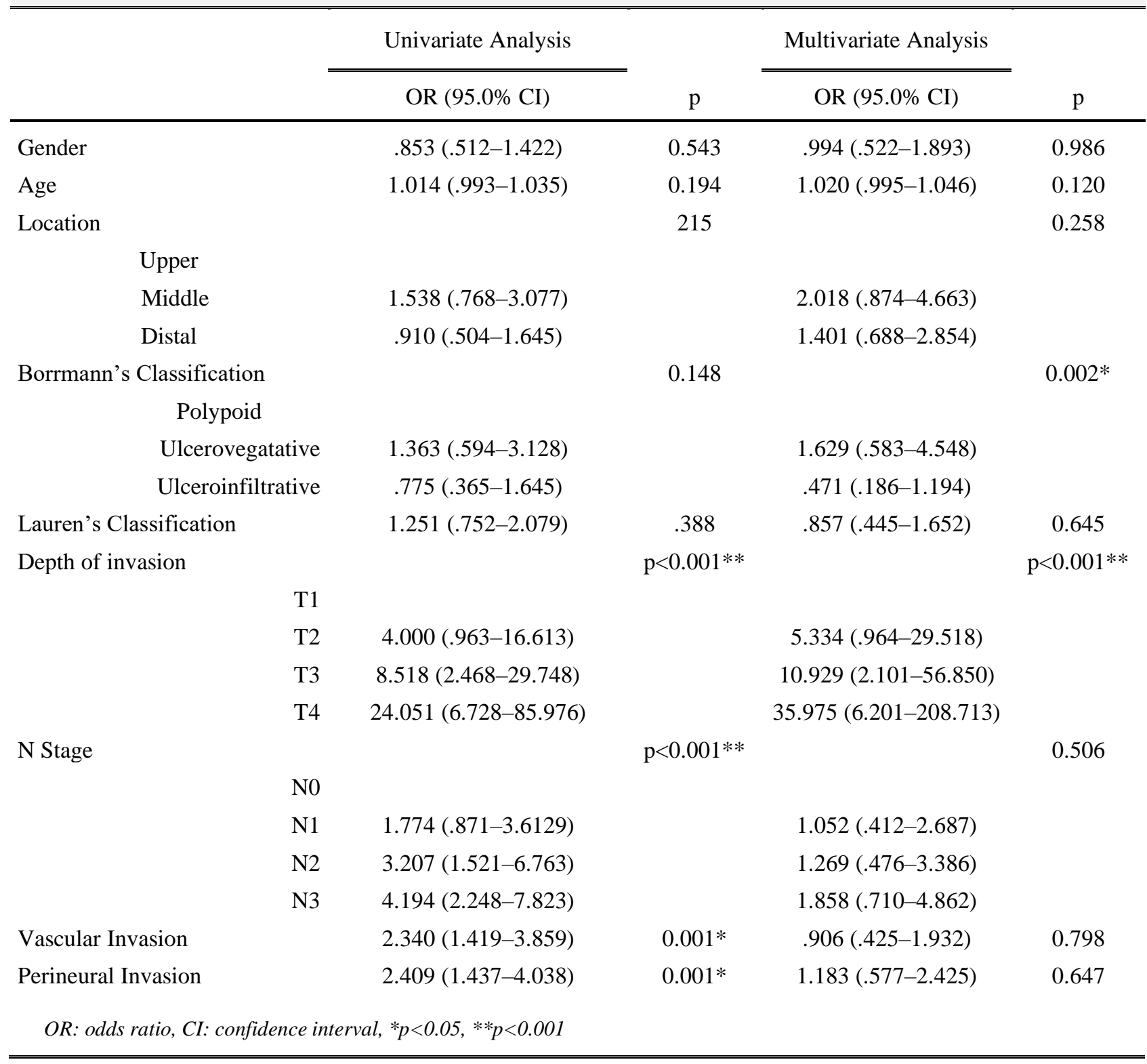

A statistical difference was noted in overall survival between the two groups $(\mathrm{p}<0.001)$ : The small-size tumor group had a mean survival time of 89 months, while the mean survival time was 62.5 months in the large-size tumor group (Figure 2, Table 3). The prognostic factors affecting survival were evaluated with univariate and multivariate stepwise Cox regression analyses for all patients (Table 4). According to the univariate analysis, the prognostic factors were tumor size $(\mathrm{p}=0.001)$, wall invasion depth $(\mathrm{p}<0.001)$, the total number of positive lymph nodes removed $(p<0.001)$, the presence of vascular invasion $(p<0.001)$ and the presence of perineural invasion; while the multivariate analysis identified prognostic factors of tumor localization $(\mathrm{p}=0.023)$, wall invasion depth $(\mathrm{p}<0.001)$, the total number of lymph nodes removed $(\mathrm{p}<0.001)$ and the total number of positive lymph nodes removed $(\mathrm{p}<0.001)$.

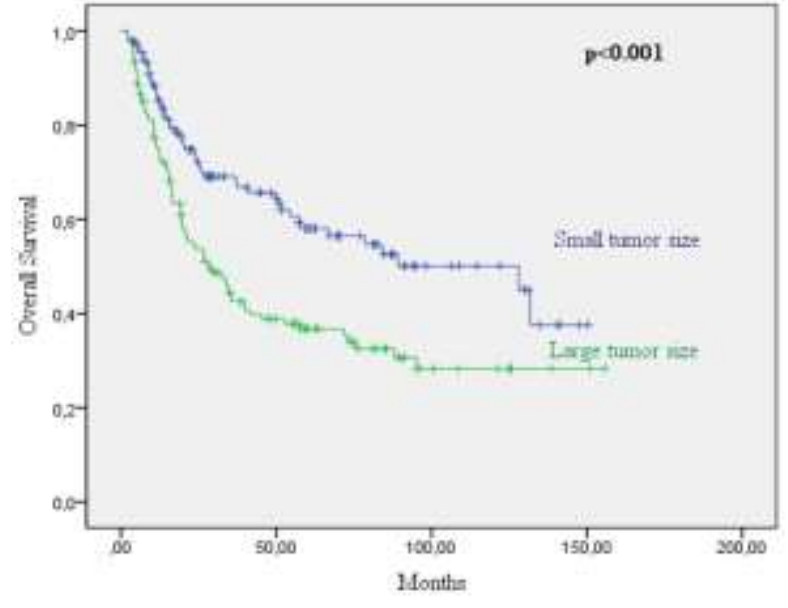

Figure 2. Overall survival comparison by tumor size (blue line: small size $\leq 4.75 \mathrm{~cm}$, green line: large size $>4.75 \mathrm{~cm}$ ) 
Table 3. Overall survival comparison by tumor size using Kaplan-Meier method

\begin{tabular}{lccc}
\hline \hline Tumor Size & \multicolumn{3}{c}{} \\
\cline { 2 - 4 } & Estimate $($ mean $) \pm$ Std. Error & $95 \%$ CI & p value \\
Small tumor size $(\leq 4.75 \mathrm{~cm})$ & $88.938 \pm 6.195$ & $76.796-101.081$ & $\mathrm{p}<0.001 * *$ \\
Large tumor size $(>4.75 \mathrm{~cm})$ & $62.614 \pm 5.885$ & $51.080-74.147$ & \\
Overall & $75.565 \pm 4.500$ & $66.746-84.385$ & \\
\hline
\end{tabular}

$* * p<0.001$, CI: Confidence Interval

Table 4. The prognostic factors for survival univariate and multivariate Cox regression analyses for all patients

\begin{tabular}{|c|c|c|c|c|c|}
\hline & & \multirow{2}{*}{$\begin{array}{c}\text { Univariate Analysis } \\
\text { OR }(95.0 \% \mathrm{CI})\end{array}$} & \multirow[b]{2}{*}{$\mathrm{p}$} & \multirow{2}{*}{$\begin{array}{l}\text { Multivariate Analysis } \\
\text { OR }(95.0 \% \mathrm{CI})\end{array}$} & \multirow[b]{2}{*}{$\mathrm{p}$} \\
\hline & & & & & \\
\hline Gender & & $.776(.535-1.126)$ & 0.182 & $1.215(.803-1.838)$ & 0.357 \\
\hline Age & & $1.007(.993-1.021)$ & 0.331 & $1.009(.993-1.025)$ & 0.278 \\
\hline Tumor size & & $1.849(1.307-2.615)$ & $0.001 *$ & $1.093(.756-1.578)$ & 0.637 \\
\hline \multirow[t]{4}{*}{ Location } & & & 0.087 & & $0.023 *$ \\
\hline & Upper & & & & \\
\hline & Middle & $1.594(.969-2.622)$ & & $1.591(.925-2.736)$ & \\
\hline & Distal & 1.069 (.681-1.676) & & $.882(.550-1.417)$ & \\
\hline Lauren's Classification & & $1.377(.952-1.993)$ & 0.089 & $.934(.626-1.395)$ & 0.738 \\
\hline \multirow[t]{5}{*}{ Depth of invasion } & & & $\mathrm{p}<0.001 * *$ & & $0.001 *$ \\
\hline & $\mathrm{T} 1$ & & & & \\
\hline & $\mathrm{T} 2$ & $3.932(.835-18.519)$ & & $4.229(.883-20.265)$ & \\
\hline & $\mathrm{T} 3$ & $7.983(1.945-32.758)$ & & $5.188(1.205-22.334)$ & \\
\hline & $\mathrm{T} 4$ & $18.820(4.602-76.957)$ & & $9.522(2.145-42.282)$ & \\
\hline Total number of lymph nodes & & $.990(.975-1.005)$ & 0.191 & $.962(.943-.981)$ & $\mathrm{p}<0.001 * *$ \\
\hline Number of positive lymph nodes & & $1.072(1.056-1.088)$ & $\mathrm{p}<0.001 * *$ & $1.082(1.057-1.108)$ & $\mathrm{p}<0.001 * *$ \\
\hline Vascular Invasion & & $2.454(1.668-3.610)$ & $\mathrm{p}<0.001 * *$ & $1.090(.707-1.682)$ & 0.695 \\
\hline Perineural Invasion & & $2.694(1.766-4.109)$ & $\mathrm{p}<0.001 * *$ & $1.307(.815-2.0959)$ & 0.267 \\
\hline \multicolumn{3}{|c|}{ OR: odds ratio, CI: confidence interval, $* p<0.05, * * p<0.001$} & & & \\
\hline
\end{tabular}

\section{Discussions}

Gastric cancer is a frequently encountered disease that is usually associated with a poor prognosis, and is among the leading causes of cancer death. It is therefore important to establish the prognostic markers for gastric cancer to improve patient survival and to identify the best treatment options for the patient [10]. The most effective prognostic factors are tumor invasion depth, lymph node involvement and metastasis status, which are used for the staging of the disease [11]. Tumor size has prognostic value in several cancer types, such as liver cancer, breast cancer and lung cancer, and is included in the TNM classification system for such cancer types; however, the role of tumor size in estimating the prognosis in gastric cancer, and whether it should be included in gastric cancer staging, are as yet uncertain [8]. Although there have been many studies reporting the independent prognostic significance of tumor size in gastric cancer, a lack of consensus remains due to the limited sample sizes and the variety of cut-off values established [8].

Del Rio P. et al. determined a cut-off value of $2.5 \mathrm{~cm}$ in their study dividing patients into small-size $(<2.5 \mathrm{~cm})$, medium-size $(2.5-5 \mathrm{~cm})$ and large-size $(>5 \mathrm{~cm})$ groups [12]. The authors found those with a tumor size of $\geq 2.5 \mathrm{~cm}$ to have a poorer prognosis, and tumor size was found to be associated with wall invasion depth and lymph node involvement [12]. In a gastric signet ring cell carcinoma study by Zhou L. et al., a cut-off value of $4.9 \mathrm{~cm}$ was identified in patients in two groups, and wall invasion 
depth and lymph node status were reported to produce differences between the groups. The large-size tumor group recorded poorer survival, while tumor size was associated with $\mathrm{T} 3, \mathrm{~T} 4 \mathrm{a}$ and $\mathrm{T} 4 \mathrm{~b}$ in a multivariate Cox regression analysis [13]. The T3-T4bN0M0 gastric cancer study by Chen $S$. et al. identified a cut-off value of $4.75 \mathrm{~cm}$ in a comparison of two groups, and reported differences in tumor localization and CEA levels between the two groups. The large-size tumor group had poorer survival, and tumor size had prognostic significance [14]. The study by Dittmar Y. et al. determined a cut-off value of $4.0 \mathrm{~cm}$ and reported differences in wall invasion depth, lymph node involvement status, lymphovascular invasion, perineural invasion, Borrmann's classification, Lauren's classification and the presence of intestinal metaplasia between the two groups [15]. The authors found survival to be poorer in gastric cancer patients with large-size tumors than in those with small-size tumors (31 months and 183 months, respectively) [15]. The ROC analysis in the present study was applied to determine the cut-off value, and resulted in a prognostic value of $4.75 \mathrm{~cm}$. A difference was noted in wall invasion depth, the number of positive lymph nodes removed, vascular invasion and perineural invasion between the two groups. A univariate analysis of tumor size identified wall invasion depth, lymph node involvement, and vascular and perineural invasion depths as prognostic risk factors, while the depth of wall invasion by the tumor and Borrmann's classification were identified as prognostic factors in the multivariate analysis. Survival was shorter in the large-size tumor group than in the small-size group (62 months vs. 88 months, respectively), and while tumor size was found to be a prognostic factor for overall survival in a univariate analysis, the multivariate analysis produced no such finding.

The limitations of the present study include its retrospective and single-center design and its small sample size.

\section{Conclusions}

As a conclusion, despite the decrease in incidence, gastric cancer is still one of the leading causes of cancer death. Tumor size is an important prognostic factor that is easy to measure endoscopically and radiologically prior to treatment planning, and can therefore be assessed as a marker in patients who cannot be assessed for tumor invasion depth and lymph node involvement for neoadjuvant therapy in the preoperative period.

\section{Conflict of interest disclosure}

There are no known conflicts of interest in the publication of this article. The study protocol was approved by the Kartal Koşuyolu High Specialty Training and Research Hospital Ethics Committee with the number 2020.4 / 26-331. A written informed consent was obtained from each participant. The study was conducted in accordance with the principles of the Declaration of Helsinki. The manuscript was read and approved by all authors.

\section{Compliance with ethical standards}

Any aspect of the work covered in this manuscript has been conducted with the ethical approval of all relevant bodies and that such approvals are acknowledged within the manuscript.

\section{Authors' contributions}

Uzun O, Senger AS, Gülmez S and Omeroglu S performed most of the study and literature search. Uzun $\mathrm{O}$, Ofluoglu $\mathrm{CB}, \mathrm{Oz}$ A designed the study and analyzed the data. Uzun O done statistical analysis, Uzun O, Senger AS, Gülmez S, wrote the manuscript, and Polat E and Duman $M$ revised the manuscript. Uzun $\mathrm{O}$ and Duman $\mathrm{M}$ approved the final version of the manuscript.

\section{References}

1. Bray F, Ferlay J, Soerjomataram I, Siegel RL, Torre LA, Jemal A. Global cancer statistics 2018: GLOBOCAN estimates of incidence and mortality worldwide for 36 cancers in 185 countries [published correction appears in CA Cancer J Clin. 2020 Jul;70(4):313]. CA Cancer J Clin. 2018;68(6):394-424. doi: $10.3322 /$ caac. 21492

2. Karimi P, Islami F, Anandasabapathy S, Freedman ND, Kamangar F. Gastric cancer: descriptive epidemiology, risk factors, screening, and prevention. Cancer Epidemiol Biomarkers Prev. 2014;23(5):700-713. doi:10.1158/1055-9965.EPI-13-1057

3. Japanese Gastric Cancer Association. Japanese gastric cancer treatment guidelines 2014 (ver. 4). Gastric Cancer. 2017;20(1):1-19. doi:10.1007/s10120-0160622-4

4. Japanese Gastric Cancer Association. Japanese gastric cancer treatment guidelines 2010 (ver. 3). Gastric Cancer. 2011;14(2):113-123. doi:10.1007/s10120011-0042-4

5. Washington K. 7th edition of the AJCC cancer staging manual: stomach. Ann Surg Oncol. 2010;17(12):30773079. doi:10.1245/s10434-010-1362-z

6. Chi Z, Huang $\mathrm{CM}$, Zheng $\mathrm{CH}$, et al. [Prognostic significance of tumor size in T3 gastric cancer]. Zhonghua Wei Chang Wai Ke Za Zhi. 2011;14(2):114116. 
7. Fukuda N, Sugiyama Y, Wada J. Prognostic factors of T4 gastric cancer patients undergoing potentially curative resection. World J Gastroenterol. 2011;17(9):1180-1184. doi:10.3748/wjg.v17.i9.1180

8. Zhao LY, Zhang WH, Chen XZ, et al. Prognostic Significance of Tumor Size in 2405 Patients With Gastric Cancer: A Retrospective Cohort Study. Medicine (Baltimore). 2015; 94(50): e2288. doi: 10.1097/MD.0000000000002288

9. Marano L, D'Ignazio A, Cammillini $\mathrm{F}$, et al. Comparison between 7th and 8th edition of AJCC TNM staging system for gastric cancer: old problems and new perspectives. Transl Gastroenterol Hepatol. 2019;4:22. doi:10.21037/tgh.2019.03.09

10. Zhang D, He W, Wu C, et al. Scoring System for Tumor-Infiltrating Lymphocytes and Its Prognostic Value for Gastric Cancer. Front Immunol. 2019;10:71. doi:10.3389/fimmu.2019.00071

11. Zhang Y, Lu JJ, Du YP, Feng CX, Wang LQ, Chen MB. Prognostic value of neutrophil-to-lymphocyte ratio and platelet-to-lymphocyte ratio in gastric cancer.
Medicine (Baltimore). 2018; 97(12): e0144. doi: 10.1097/MD.0000000000010144

12. Del Rio P, Viani L, Bertocchi E, et al. The prognostic role of tumor size in patients with gastric cancer. Ann Ital Chir. 2017;88:478-484.

13. Zhou L, Li W, Cai S, Yang C, Liu Y, Lin Z. Large tumor size is a poor prognostic factor of gastric cancer with signet ring cell: Results from the surveillance, epidemiology, and end results database. Medicine (Baltimore). 2019;98(40):e17367. doi:10.1097/MD.0000000000017367

14. Chen S, Ou-Yang LY, Nie RC, et al. Tumor Size Is a Critical Factor in Adjuvant Chemotherapy for T34aN0M0 Gastric Cancer Patients after D2 Gastrectomy. Gastroenterol Res Pract. 2017;2017: 4928736. doi:10.1155/2017/4928736

15. Dittmar Y, Ardelt M, Scheuerlein H, Rauchfuss F, Dondorf $F$ and Settmacher U. The Impact of Tumor Size on the Prognosis of Gastric Cancer: Experiences from a European Study Group. Austin J Surg. 2016; 3(1): 1082. 